F. Liberati, A. Di Giorgio, A. Giuseppi, A. Pietrabissa and F. Delli Priscoli, "Efficient and Risk-Aware Control of Electricity Distribution Grids," in IEEE Systems Journal.

doi: 10.1109/JSYST.2020.2965633

URL:

http://ieeexplore.ieee.org/stamp/stamp.jsp?tp=\&arnumber=8966461\&isnumber=4357939

Copyright notice:

(C) 2020 IEEE. Personal use of this material is permitted. Permission from IEEE must be obtained for all other uses, in any current or future media, including reprinting/republishing this material for advertising or promotional purposes, creating new collective works, for resale or redistribution to servers or lists, or reuse of any copyrighted component of this work in other works. 


\title{
Efficient and Risk-Aware Control of Electricity Distribution Grids
}

\author{
Francesco Liberati ${ }^{\circledR}$, Member, IEEE, Alessandro Di Giorgio ${ }^{\circledR}$, Member, IEEE, \\ Alessandro Giuseppi $\left.{ }^{(}\right)$Member, IEEE, Antonio Pietrabissa ${ }^{(}$, Member, IEEE, and \\ Francesco Delli Priscoli ${ }^{\circledR}$, Member, IEEE
}

\begin{abstract}
This article presents an economic model predictive control (EMPC) algorithm for reducing losses and increasing the resilience of medium-voltage electricity distribution grids characterized by high penetration of renewable energy sources and possibly subject to natural or malicious adverse events. The proposed control system optimizes grid operations through network reconfiguration, control of distributed energy storage systems (ESSs), and on-load tap changers. The core of the EMPC algorithm is a nonconvex optimization problem integrating the ESSs dynamics, the topological and power technical constraints of the grid, and the modeling of the cascading effects of potential adverse events. An equivalent (i.e., having the same optimal solution) proxy of the nonconvex problem is proposed to make the solution more tractable. Simulations performed on a 16-bus test distribution network validate the proposed control strategy.
\end{abstract}

Index Terms-Energy storage systems (ESSs), model predictive control (MPC), network reconfiguration, resilient control, smart grids.

\section{NOMENCLATURE}

Indices and Sets

$k \quad$ Current time.

$h \quad$ Time index in the control window, $h \in[k, k+$

$N-1]$.

$i, j \quad$ Bus indices.

$\mathcal{V}^{\text {ESS }} \quad$ Set of network nodes hosting ESSs.

$\mathcal{V}^{\text {DG }} \quad$ Set of network nodes hosting DGs.

$\mathcal{V}^{\mathrm{SB}} \quad$ Set of HV/MV substations.

\section{Parameters}

$\mathcal{A} \quad$ Set of network lines.

$B_{i j} \quad$ Susceptance of line $(i, j)$.

$C_{i}^{\text {ESS }} \quad$ Capacity (kWh) of the ESSs at bus $i$.

$G_{i j} \quad$ Conductance of line $(i, j)$.

$N \quad$ Length of the prediction horizon.

$r_{i}, r_{i j} \quad$ Risk value associated to bus $i$ and line $(i, j)$.

Manuscript received April 19, 2019; revised November 20, 2020 and January 3, 2020; accepted January 4, 2020. This work was supported by the ATENA project, which has received funding from the European Union's Horizon 2020 research and innovation programme under grant agreement No 700581. (Corresponding author: Francesco Liberati.)

The authors are with the DIAG Department, Sapienza University of Rome, 00185 Rome, Italy, and also with CyberSecurity Research Group, CRAT, 00195 Rome, Italy (e-mail: liberati@diag.uniroma1.it; digiorgio@diag.uniroma1.it; giuseppi@diag.uniroma1.it; pietrabissa@diag.uniroma1.it; dellipriscoli@diag. uniroma1.it).

Digital Object Identifier 10.1109/JSYST.2020.2965633
$\mathrm{SOC}_{i}^{\mathrm{ref}} \quad$ Reference state of charge of the ESS at bus $i$.

$S, Z \quad$ Auxiliary super source and super sink nodes.

$T \quad$ Sampling period.

$\mathcal{V} \quad$ Set of network nodes.

$V_{i j}^{\text {OLTC }} \quad$ OLTC voltage level at node $i$ for turn ratio $j$.

$\mathbf{x}_{\mathbf{k}} \quad$ Vector gathering the state feedback at time $k$.

$Y_{i j} \quad$ Line series admittance.

$\alpha, \beta, \gamma, \delta, \epsilon$ Weight parameters of the objective function.

Control Variables

$a_{i j}(h) \quad$ Connection status of line $(i, j)$ at time $h$.

$P_{i}^{\mathrm{ESS}}, Q_{i}^{\mathrm{ESS}}$ Active/reactive power of the ESS at bus $i$.

$\mathbf{u}_{\mathbf{k}} \quad$ Vector of the control variables over the control window.

$\delta_{i j} \quad$ Boolean variable indicating the OLTC level selected at (substation) bus $i$.

$\sigma \quad$ Load shedding factor.

Other Variables

$\mathcal{F} \quad$ Target function.

$C_{i} \quad$ Centrality of node $i$.

$f_{i, j}, f_{i, j}^{c} \quad$ Radiality and Centrality flux in line $(i, j)$.

$I_{i, j} \quad$ Current flowing in line $(i, j)$.

$P_{i}, Q_{i} \quad$ Active/reactive power injected at bus $i$.

$P_{i j}, Q_{i j} \quad$ Active/reactive power injected into line $(i, j)$.

$P_{i}^{\mathrm{DG}}, Q_{i}^{\mathrm{DG}} \quad$ Active/reactive power generated by the DG at bus $i$.

$P_{i}^{\text {load }}, Q_{i}^{\text {load }}$ Active/reactive load at bus $i$.

$P_{i j}, Q_{i j} \quad$ Active/reactive power flows from node $i$ to node $j$.

$R_{i j} \quad$ Auxiliary variable

$\mathrm{SOC}_{i} \quad$ State of charge of the ESS at bus $i$.

$T_{i j} \quad$ Auxiliary variable.

$u_{i} \quad$ Auxiliary variable.

$V_{i} \quad$ Voltage magnitude at bus $i$.

$\theta_{i}, \theta_{i j} \quad$ Voltage angle at bus $i, \theta_{i j}=\theta_{i}-\theta_{j}$.

min max Limit values of the corresponding variable.

Acronyms

DG Distributed generation.

EMPC Economic model predictive control.

ESS Energy storage system.

HV High voltage.

LV Low voltage.

MPC Model predictive control.

MV Medium voltage.

OLTC On-load tap changer. 


$\begin{array}{ll}\text { OPF } & \text { Optimal power flow. } \\ \text { PV } & \text { Photovoltaic. } \\ \text { RES } & \text { Renewable energy sources. } \\ \text { SAIDI } & \text { System average interruption duration index. } \\ \text { SAIFI } & \text { System average interruption frequency index. } \\ \text { SCADA } & \text { Supervisory control and data acquisition. } \\ \text { SOC } & \text { State of charge. }\end{array}$

\section{INTRODUCTION}

\section{A. Background and Aim}

E LECTRICITY distribution networks are cyber-physical systems that work based on the interplay of the physical grid, characterized by its constraints and dynamics, and dedicated information and communication technology systems (supervisory control and data acquisition, energy management systems, etc.). The transition toward distributed generation (DG) and electro-mobility is making the operation of the electricity network more complex [1], [2]; at the same time, digital innovations potentially introduce new vulnerabilities and raise concerns for cyber security [3]. This article proposes a model predictive control (MPC) strategy for minimizing network losses and the impact of adverse events in medium-voltage (MV) distribution grids characterized by high renewable energy sources (RES) penetration. This is achieved by coordinating the control of line switches (which allow to reconfigure the topology of the grid), the on-load tap changer (OLTC) deployed at the level of high voltage (HV) to MV substations (which enable voltage control), and the distributed energy storage systems (ESS). Three main use cases are discussed (see Section II): 1) power losses minimization during normal grid operation; 2) risk-aware grid operation, in case information about the operative level of the different subsystems of the grid is available; and 3) resilient grid control in case of loss of power at the HV/MV primary substations.

\section{B. Related Works}

Although the problem of ensuring an adequate level of security against cyber-physical attacks is relatively recent, the one of efficiently operating the electricity distribution grid has been largely studied in the technical literature, typically considering the computation of optimal network configurations minimizing the network power losses. Recent relevant papers dealing with network reconfiguration and at the base of this work are [4][13]. In [4] and [5], a network reconfiguration algorithm was presented to minimize power losses in a scenario foreseeing high penetration of DG. In particular, Jabr et al. [4] presented an exact, nonconvex and nonlinear formulation of the problem and show how, based on a load-flow technique introduced in [6], the nonconvex formulation can be turned into an equivalent convex one, more suitable for online applications, such as the one in this article. A similar convexification procedure, presented in [14], is used in [5] for the relaxation of the nonlinearities of the power flow equations. In [7] instead, a coordinated control for voltage regulation and network reconfiguration was proposed. The present article extends the contributions in [4], [5], and [7] by introducing the ESS control in combination with the network reconfiguration and OLTC control, and by extending the formulation to the multitime slot case, utilizing the MPC approach and, thus, providing predictive capabilities to the control strategy.

In [9], Muhtazaruddin et al. jointly consider network reconfiguration and optimal placement and sizing of DG and capacitors, via an artificial bee colony optimization approach. A similar placement and sizing optimization problem is solved for ESS and DG in [15], which proposes yearly reconfiguration to increase the network efficiency and optimally plan upgrading interventions. The minimization of operative costs and power losses is proposed in [15], by defining a stochastic mixed integer linear programming problem that clusters demand-generation patterns into so-called snapshots. Another recent work that deals with both optimal ESS placement and efficient network operation is [10], where Bai et al. combined the problem of planning ESS placement and sizing with an hourly network reconfiguration in order to minimize power losses. Differentiating from the above works, this article deals with online optimization (every $15 \mathrm{~min}$ ) of network operation, to increase both the efficiency and the resiliency of the network in response to real-time forecasts of load and generation profiles.

Another key reference for the present article is [16], which shows that the network radiality and the connectivity of loads to HV/MV substations - two typical requirements in the operation of distribution grids - can be guaranteed, in passive networks, by combining a topological constraint on the number of active lines with the power flow equations (see Section III). This is, however, not sufficient to guarantee radiality in active networks, hosting energy sources, such as ESS and DG [17]. A work around in [16] is to add fictitious loads at the DG nodes and impose their connection to the substation via additional constraints. The present article extends [16] by introducing a first set of conditions to enforce radiality, and a second set of conditions to capture specific topological properties of the network configuration, such as hop distance from the substation, which allows to model aspects such as cascading effects and interdependencies in the optimization problem. As a result, the proposed control strategy will not give rise to loops in the network and/or islanded configurations during normal operation, unless explicitly allowed via the selection of specific control parameters (see Section II-A3).

Also, heuristic methods have been proposed for network reconfiguration. In [18], Chang et al. employed colored Petri networks to develop a set of reconfiguration rules; in [19], a runner-root algorithm is developed to reduce power losses and balance the loads in the network; in [20], a genetic algorithm is presented to solve the optimal allocation of DG and the network reconfiguration problem.

Finally, two other relevant works related to the present article are [12] and [13], which propose network reconfiguration to minimize grid losses and optimize grid reliability. Specifically, the latter is achieved via the optimization of network reliability indicators such as the system average interruption duration index and the system average interruption frequency index [21], based on the knowledge of historical data. The present article extends the approach in [12] to the real-time optimization of network risk, by introducing the reaction to notification alerts coming from a risk predictor system (see Section II), without relying on heuristic solutions and while coordinating the reconfiguration with ESS control. 


\section{Main Contributions}

The main contributions and the distinctive features of this article are as follows.

1) The formulation of a joint network reconfiguration, ESS and OLTC control problem, for secure and efficient operation of the grid.

2) The introduction of a multitime slot control horizon, which allows us to tackle dynamic scenarios characterized by variable power injections from RES and variable operative levels of network components.

3) The use of the MPC concept, which enables real-time reaction to updates of short-term RES forecasts and notifications of adverse events, while taking into account economic aspects of the network operation and keeping the ESS operation feasible.

4) The application of the method presented in [6] for building a conified proxy of the nonconvex optimization problem at the basis of MPC, rising from the inclusion of the hybrid power flow equations in the reconfiguration problem.

5) The introduction of a set of fully topological constraints to guarantee network radiality and avoid network islanding, in networks with DG and ESSs.

6) The integration (in addition to the "classical" power losses minimization terms) of specific terms related to the minimization of the impact arising from malicious/adverse events and/or the degraded operative level of grid components. This establishes a real-time tradeoff between the requirement of efficient operation and that of risk minimization (the topological constraints allows us to model cascading effects and, hence, to compute risk metrics associated to the network configuration).

7) The support of grid islanding operation in absence of main supply from the transmission network. The characterizing aspect is the efficient use of the ESSs to maximize grid survivability. This is achieved by dynamically changing the net load sustained by the ESSs, through the proper shift of load and DG among network trees via reconfiguration and load shedding.

\section{Article Organization}

Section II presents the reference scenario, the addressed use cases, and the logic underlying the proposed control system. Section III details the mathematical formulation of the proposed control problem. Section IV discusses simulation results. Section V presents conclusions and future research.

\section{REFERENCE SCENARIO AND CONTROL LOGIC}

\section{A. Reference Scenario and Use Cases}

The reference scenario discussed in this article considers the control of a reconfigurable MV grid equipped with storage and DG units. The controlled elements include: the ESS (to inject/adsorb power for balancing purposes), the OLTC (with effect on voltage control), the switches (for grid topology reconfiguration), and sheddable loads (in case of islanding operations). The control system proposed in the following is designed focusing particularly on the following use cases.

1) Minimization of Power Losses Against RES Fluctuations: This is the base use case aimed at mitigating the impact of time-varying and potentially volatile generation and consumption patterns on the grid in terms of network power losses. Day-ahead and short-term predictions of DG are supposed to be available to the control system, which reconfigures the grid and controls ESSs/OLTC in real time to achieve the objective.

2) Preventive Mitigation of Adverse Events in the Distribution Grid: This use case focuses on risk-aware grid operation, in which the grid is operated taking into account current and future estimated risk levels of its components. In details, the current and predicted risk levels related to network nodes and lines are assumed to be available to the controller from a risk predictor system, which correlates heterogeneous information (e.g., ongoing anomalies, cyber-attacks detected, and meteorological conditions) regarding the state of the infrastructure, and computes a short-term prediction of the risk level associated with the various system components (see [22] and [23] for details on the functioning of the risk predictor, which has been developed and demonstrated in the context of the European project ATENA). Risk levels are associated to both lines and nodes of the grid, in order to capture different kinds of adverse events or cyber-attacks.

3) Preventive Mitigation of Adverse Events at Transmission/Distribution Interface: This use case concerns adverse events impacting on the transmission network and propagating to the distribution side, like cyber-attacks against large power plants and HV/MV substations, which can lead to the disconnection of the distribution side from the transmission network and give rise to islanded operation. In this case, grid loading, voltage, and frequency control [24] are sustained by the DG and the ESSs. The control objective is to maximize the survivability of the distribution grid by properly operating DG resources and the ESS, and resorting to load shedding only to increase the survival time of the network.

\section{B. Control Methodology and Application Logic}

The block diagram of the controlled system is presented in Fig. 1 and explained in details in this and the next section. Dealing at the same time with efficiency and security-related aspects, the most natural choice to address the problem is the implementation of a predictive controller, which shall take its control actions considering also the future effects that these will have on the system, in order to prevent unsafe operations and optimize efficiency in the long term. A promising solution is represented by economic MPC (EMPC), a variant of MPC that explicitly takes into account the optimization of system performance and efficiency metrics (see, e.g., [25] and [26]). In MPC [27], at the generic time $k$, input data (short term $\mathrm{load} / \mathrm{generation}$ forecasts and predicted risk values in the present case) and the feedback of the state of the plant (step 1 in Fig. 1) are retrieved, and the control signals are computed by solving a constrained optimization problem defined over a time window $N$ steps in the future (step 2); the first sample of the computed control signals is applied to the plant (step 3) and, then, the process is reiterated at time $k+1$.

The iterative repetition of the optimization procedure, referred to in the literature as "receding horizon paradigm," is the fundamental characteristic of MPC control, and represents its main advantage over other control schemes. Taking, at every time step $k$, a state feedback from the system, and consequently updating 


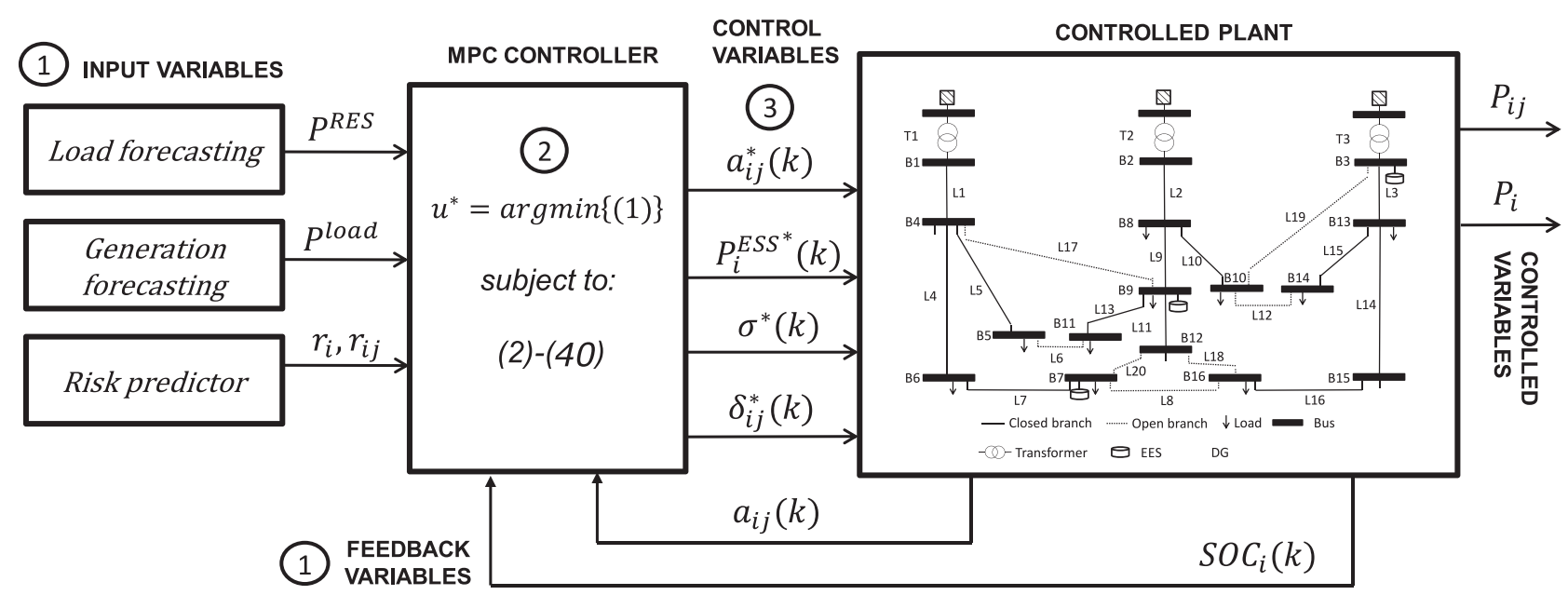

Fig. 1. Block diagram of the proposed control system.

the optimal control trajectory, may be seen as bringing the intrinsic properties of robustness that characterize closed-loop control into the optimization domain that often characterizes open-loop controllers. Being the system considered in this article characterized by significant uncertainties on the long-term behavior of some of its components (e.g., photovoltaic (PV) plants and load profiles), applying such a scheme represents an ideal solution with respect to other optimization approaches that assume a perfect knowledge of the system, or at least of its stochastic behavior.

In EMPC, the objective function is selected to optimize the performance of the system. In this article, it is used to jointly account for economic, security, and stability requirements. The computed control signals are the state of the switches, the ESS charging/discharging power, and the position of the OLTCs. The feedback acquired at $k$ is given by the state of the controlled switches and the state of charge (SOC) of the ESS.

\section{MAthematical Formulation OF THE CONTROL PROBLEM}

We discuss in the following the optimization problem that is solved at each time $k$ by the MPC controller (step 2 in Fig. 1). The problem is defined over the time interval $[k, k+N-1]$, referred to as control horizon. In the following, a second time index $h \in[k, k+N-1]$ is used to denote the generic time instant within the control horizon. Regarding the main nomenclature used, as customary in network reconfiguration studies, the topological aspect of the distribution network is modeled by a graph $\mathcal{G}=\{\mathcal{V}, \mathcal{A}\}$, where $\mathcal{V}$ is the set of network buses and $\mathcal{A}$ is an adjacency matrix specifying the physical connections, i.e., $a_{i j}=a_{j i}=1$ if there exists a line between buses $i$ and $j$, zero otherwise. The Boolean control variable $a_{i j}(h)$ captures the status (connected/disconnected) of line $(i, j)$ at time $h$ (the status can change over time only for the subset of lines that can be switched). In the following, the standard notation adopted in power system studies is used: $P_{i}$ is the bus power injection (positive) or withdrawal, $P_{i j}$ and $Q_{i j}$ denote, respectively, the active and reactive line flows, $V_{i}$ is the bus voltage, $\theta_{i}$ is the bus voltage angle, and $\theta_{i j}$ is the difference between voltage angles at buses $i$ and $j$. Power injection and withdrawal at the different bus types are specialized by the use of acronyms in superscript $\left(P_{i}^{\mathrm{DG}}\right.$, $P_{i}^{\mathrm{ESS}}, P_{i}^{\text {load }}$, etc.). Finally, additional specific notation is defined in the following in the place where it is introduced (all symbols are as well defined in the nomenclature section). The objective function and the constraints are presented and discussed in the following.

1) Objective Function: The objective function is designed to jointly optimize the performance of the system and its resilience to faults/attacks, in a multiobjective sense. It is given by five main terms, each weighed by the coefficients $\alpha, \beta, \gamma, \delta, \epsilon$

$$
\begin{aligned}
& \mathcal{F}_{N}\left(k, \mathbf{x}_{k}, \mathbf{u}_{k}\right)=\sum_{h=k}^{k+N-1}\left\{\alpha(h) \sum_{i \in \mathcal{V}} P_{i}(h)+\beta(h)\right. \\
& \times \sum_{i, j \in \mathcal{V}, j>i}\left[a_{i j}(h)\left(1-a_{i j}(h-1)\right)+a_{i j}(h-1)\left(1-a_{i j}(h)\right)\right] \\
& +\gamma(h) \sum_{i \in \mathcal{V}}\left[C_{i}(h) r_{i}(h)+\sum_{j \in \mathcal{V}: j>i} a_{i j}(h) r_{i j}(h)\right] \\
& \left.+\delta(h) \sum_{i \in \mathcal{V} \text { ESS }}\left[\operatorname{SOC}_{i}(h)-\operatorname{SOC}_{i}^{\mathrm{ref}}\right]^{2}+\epsilon(h) \sigma(h)\right\}
\end{aligned}
$$

The subscript $N$ in $\mathcal{F}_{N}\left(k, \mathbf{x}_{k}, \mathbf{u}_{k}\right)$ is to denote that the optimal control problem is defined over $N$ time intervals, and $\mathbf{x}_{k}$ denotes the state of the controlled elements at time $k$, as acquired via feedback from the controlled devices. It is given by the state of the switches and the SOC of the ESS, i.e., $\mathbf{x}_{k}:=\left\{a_{i j}(k),(i, j) \in \mathcal{A}\right\} \cup\left\{\operatorname{SOC}_{i}(k), i \in \mathcal{V}^{\mathrm{ESS}}\right\} . \mathbf{u}_{k}$ is the set of decision variables, i.e., the switching actions, the position of the tap changers, the ESS power injections, and the load shedding (only in the islanded case), i.e., $\mathbf{u}_{k}:=\left\{a_{i j}(h)\right.$, $(i, j) \in \mathcal{A}, \quad h \in[k, k+N-1]\} \cup\left\{P_{i}^{\mathrm{ESS}}(h), \quad i \in \mathcal{V}^{\mathrm{ESS}}, h \in\right.$ $[k, k+N-1]\} \cup\{\sigma(h), h \in[k, k+N-1]\}$. Each term of the target function is explained in the following. The term 
$\mathcal{F}_{1}:=\sum_{i \in \mathcal{V}} P_{i}(h)$ represents the network power losses foreseen at time $h$ (given by the sum of the power injected/withdrawn at the grid buses). The term $\mathcal{F}_{2}:=\sum_{i \in \mathcal{V}} \sum_{j \in \mathcal{V}: j>i}\left[a_{i j}(h)(1-\right.$ $\left.\left.a_{i j}(h-1)\right)+a_{i j}(h-1)\left(1-a_{i j}(h)\right)\right]$ is the number of switching operations at $h$. Minimizing $\mathcal{F}_{2}(k)$ avoids unnecessary switching actions. The term $\mathcal{F}_{3}:=\sum_{i \in \mathcal{V}}\left[C_{i}(h) r_{i}(h)+\right.$ $\left.\sum_{j \in \mathcal{V}: j>i} a_{i j}(h) r_{i j}(h)\right]$ enables risk-aware optimization by including the risk levels of nodes and lines (available from the risk predictor). This is particularly relevant in scenarios in which components' operative levels are impacted by adverse events. Parameters $r_{i j}(h), r_{i}(h) \in[0,1]$ are the predicted "risk levels" for the generic line $(i, j)$ and for node $i$ at $h$, respectively. They can be understood as the 1-complement of the operative level or the availability of a given subsystem, so that the higher $r_{i j}(h), r_{i}(h)$, the higher is the probability that the given system will experience a malfunctioning at time $h$. The role of variable $C_{i}(h)$ is to capture a centrality measure of the generic node $i$, i.e., the number of nodes that would lose their connection to the primary substation in case of failure of node $i$. The product $C_{i}(h) r_{i}(h)$, hence, provides a measure that is proportional to the expected number of nodes that will be disconnected after the failure of node $i$, given the network configuration at time $h$. It is worth remarking that the centrality has to be computed online during the optimization, through the inclusion of a specific set of constraints, detailed in the following. According to the proposed definition, $C_{i}(h)$ will be proportional to the square of the number of descendant nodes, enhancing the controller sensitivity to higher risk levels. The second term in $\mathcal{F}_{3}$ is included in order to penalize the usage of links characterized by high risk levels. The term $\mathcal{F}_{4}:=\sum_{i \in \mathcal{V}^{\mathrm{ESS}}}\left[\mathrm{SOC}_{i}(h)-\mathrm{SOC}_{i}^{\mathrm{ref}}\right]^{2}$ is a regulation term, which ensures that the ESS are operated near a reference SOC, in order to maintain absorption/injection control margins. The term $\mathcal{F}_{5}:=\sigma(h)$ is a load shedding factor, which is considered only in the islanded operation, when load is potentially shed in order to further prolong the operation of the network (a uniform shedding factor $\sigma(h) \in[0,1]$ is considered, so to keep the discussion simple-more refined shedding criteria can be easily integrated).

The weights $\alpha(h), \beta(h), \gamma(h), \delta(h), \epsilon(h)$ determine the tradeoff among the various objectives. Their time dependence is due to the fact that they may also weight differently short-term versus long-term performances: due to the uncertainty affecting the prediction of the exogenous signals, it may be reasonable to give priority to objectives closer in the prediction window, as the controller can assume a higher confidence level on the short-term predictions. Tuning of the weights can be done based on economic considerations: $\alpha$ can be chosen as the cost of system losses, $\beta$ as the cost of the single switching operation (e.g., total cost of device over the number of guaranteed switching operations over the life time), $\gamma$ based on cost of risk, risk aversion considerations, and $\epsilon$ the cost of shedding power (while $\delta$ has an inherently technical meaning). Another practical approach to tuning is to sample the space of the coefficients of the target function and perform simulations, in representative scenarios, for each configuration of the parameters. Then, for each simulation, resulting key performance indicators of interest for the operator (like, number of switching operations, total system losses, risk level, etc.), are reported on the same graph, which will typically have a Pareto-like shape. This graph can be used by the operator as a "tuning chart" to help him/her select good configuration of the coefficients depending on the desired optimization goals.

The other fundamental parameter that defines the overall controller is the length of the prediction horizon, $N$, that should never be longer than the confidence interval over which the predictions provided to the control system can be assumed to be reasonably accurate. For this reason, in our testing, we set the window to be 3 -h long.

2) Constraints: The following constraints are included, defined for $h \in[k, k+N-1]$.

a) Power flow constraints: The real and reactive power flows from node $i$ to node $j$ are (see, e.g., [6])

$$
\begin{aligned}
P_{i j}(h)=a_{i j}(h)[ & -G_{i j} V_{i}(h) V_{j}(h) \cos \left(\theta_{i j}(h)\right) \\
& \left.-B_{i j} V_{i}(h) V_{j}(h) \sin \left(\theta_{i j}(h)\right)+G_{i j} V_{i}^{2}(h)\right] \\
Q_{i j}(h)=a_{i j}(h)[ & +B_{i j} V_{i}(h) V_{j}(h) \cos \left(\theta_{i j}(h)\right) \\
& \left.-G_{i j} V_{i}(h) V_{j}(h) \sin \left(\theta_{i j}(h)\right)-B_{i j} V_{i}^{2}(h)\right]
\end{aligned}
$$

where $\theta_{i j}(h)=\theta_{i}(h)-\theta_{j}(h)$. Equations (2) and (3) are defined for $(i, j): a_{i j}=1 . G_{i j}$ and $B_{i j}$ are, respectively, the line series conductance and susceptance, i.e., the real and the imaginary parts of the $(i, j)$ line series admittance $Y_{i j}$. Lines' shunt elements are neglected (a reasonable assumption in distribution networks [28]).

b) Power balance equations: For all $i \in \mathcal{V}$

$$
\begin{aligned}
P_{i}(h) & =P_{i}^{S B}(h)+P_{i}^{D G}(h)+P_{i}^{\mathrm{ESS}}(h)-P_{i}^{\text {load }}(h) \\
& =\sum_{j} P_{i j}(h)+G_{i i}\left(V_{i}(h)\right)^{2} \\
Q_{i}(h) & =Q_{i}^{S B}(h)+Q_{i}^{D G}(h)+Q_{i}^{\mathrm{ESS}}(h)-Q_{i}^{\text {load }}(h) \\
& =\sum_{j} Q_{i j}(h)-B_{i i}\left(V_{i}(h)\right)^{2} .
\end{aligned}
$$

Depending on the bus type, one or more terms might be zero.

c) Radiality constraints: In the far majority of cases, distribution networks are radially operated. Both heuristic and exact radiality constraints have been proposed in the literature, but, as discussed, most of them work only in case of passive networks [11]. Lavorato et al. [16] and [17] have recently proposed conditions to ensure radiality in active distribution grids. Based on [16], the following constraint is included:

$$
\sum_{\left\{i j: a_{i j}=1, j>i\right\}} a_{i j}(h)=|\mathcal{V}|-\left|\mathcal{V}^{\mathrm{SB}}\right|
$$

which states that the number of connected lines in the network shall equal the number of nodes minus the number of HV/MV substations. In passive networks, (6) combined with (4) and (5) guarantees radiality [17]. In active networks with ESS, (6) ensures radiality only if the ESS are hosted at primary substation level (otherwise the formation of islands powered by the ESSs is possible), as shown in [17]. In general, this kind of constraints avoid the presence of islanded portions of the network exploiting the unfeasibility of their power flow, which in active networks, due to the presence of devices such as ESS, is no longer a correct assumption. In the following, 
(6) is extended in order to guarantee radiality in the general active networks case. An auxiliary flux is introduced to ensure that each ESS is connected to an HV/MV substation, similarly to what presented in [16]. This connectivity flux goes from the transmission network into the distribution network, and is forced to reach all the ESSs, assuring hence the connectivity sought. To formalize this auxiliary flux, two auxiliary nodes are added: a super source $S$, representing the transmission network, connected to each HV/MV substation through outgoing edges, and a super sink $Z$, connected to each ESS through incoming edges. In addition, two auxiliary flux variables $f_{i j}$ and $f_{j i}$ are associated to each link in the augmented network, representing the link flows in the two opposite directions. The following constraints, modeling flux conservation for nodes and capacity limits for links, guarantee the connectivity desired for the ESSs:

$$
\begin{aligned}
& f_{i j}(h) \in\left\{0,1, \ldots,\left|\mathcal{V}^{\mathrm{ESS}}\right|\right\} \quad \forall i, j \in \mathcal{V} \cup\{S, Z\} \\
& a_{i j}(h) \geq \frac{f_{i j}(h)+f_{j i}(h)}{\left|\mathcal{V}^{\mathrm{ESS}}\right|} \quad \forall i, j \in \mathcal{V} \cup\{S, Z\} \\
& f_{i j}(h) f_{j i}(h)=0 \quad \forall i, j \in \mathcal{V} \cup\{S, Z\} \\
& \sum_{j \in \mathcal{N}_{i}(h)} f_{i j}(h)-\sum_{j \in \mathcal{N}_{i}(h)} f_{j i}(h)=0 \quad \forall i \in \mathcal{V} \\
& \sum_{j \in \mathcal{N}_{S}(h)} f_{S j}(h)=\left|\mathcal{V}^{\mathrm{ESS}}\right| \\
& f_{j Z}(h)=1 \quad \forall j \in \mathcal{N}_{Z}
\end{aligned}
$$

where $\left|\mathcal{V}^{\mathrm{ESS}}\right|$ is the number of buses hosting ESS and $\mathcal{N}_{i}$ represents the neighbors of the node $i$ (i.e., $\mathcal{N}_{S}$ are the HV/MV substations and $\mathcal{N}_{Z}$ the buses hosting ESS). Constraint (9) prevents from having flows in both directions on each link. Constraint (11) states that the flow from the super source has to be equal to $\left|\mathcal{V}^{\mathrm{ESS}}\right|$, while (12) forces the connection with an $\mathrm{HV} / \mathrm{MV}$ substation for all the ESS and (10) models the flux conservation. Constraint (8) forces $a_{i j}=1$ if any flux passes through branch $(i, j)$ in either direction. A possible interpretation of constraints (7)-(12) is that they force the existence of a path between the HV/MV substations and the ESSs by the mass/flux conservation law, ensuring their connection. Since the ESSs are now always connected to the transmission line, they cannot act as a slack node for an independent island, whose presence could imply the nonradiality of the network [17], and constraint (6) returns a valid mean to impose radiality.

d) Definition of $C_{i}(h)$ in $(1)$ : Variable $C_{i}(h)$ should be informative of the number of nodes that are the descendants of a given node $i$ at time $h$ from a topological point of view. In general, this variable can be computed in polynomial time on a fixed network configuration, but, in order to be included in the optimization process, for the dynamic network reconfiguration problem, it has to be computed by the optimization solver for each of the topologies it evaluates. For this reason, a second auxiliary flow is introduced in the following. This new flux is assumed to be produced by the super source node $S$, and absorbed by the super sink node $Z$. The idea is that each node shall feed exactly one unit of flow to $Z$, meaning that the intake of flux that the node $i$ receives is exactly the number of its topological descendants, increased by one. To attain this result, all the nodes in the network are connected to the super sink through outgoing edges with unitary capacity. $S$ feeds the HV/MV substations with a total quantity of flux equal to the number of buses. Let $f_{i j}^{c}(h)$ represent the flow in the link connecting the nodes $i$ and $j$ : $f_{i j}^{c}(h) \in\left\{-\left|\mathcal{V}^{\mathrm{SB}}\right|, \ldots, 0, \ldots,\left|\mathcal{V}^{\mathrm{SB}}\right|\right\} \quad \forall i, j \in V \cup\{S, Z\}$. The following constraints are then included:

$$
\begin{aligned}
& \sum_{j \in \mathcal{N}(S)} f_{S j}^{c}(h)=\left|\mathcal{V}^{\mathrm{SB}}\right| \\
& f_{j Z}^{c}(h)=1 \quad \forall j \in \mathcal{N}(Z) \\
& a_{i j}(h) \geq\left(\frac{f_{i j}^{c}(h)}{\left|\mathcal{V}^{S B}\right|}\right)^{2} \forall i, j \in V \cup\{S, Z\} \\
& \sum_{j \in \mathcal{N}(i)} f_{i j}^{c}(h)-\sum_{j \mid i \in \mathcal{N}(j)} f_{j i}^{c}(h)=0 \quad \forall i \in \mathcal{V} \\
& C_{i}(h) \geq \sum_{j \in \mathcal{N}(j)} f_{i, j}^{c}(h)^{2}+\sum_{j \mid i \in \mathcal{N}(j)} f_{j, i}^{c}(h)^{2} \quad \forall i \in \mathcal{V} .
\end{aligned}
$$

Notice that in this case, the physical links are given an arbitrary direction on the flow graph, as there is no equivalent constraint to (9) and $f_{i j}^{c}$ can assume also negative values. To address this change, (8) has been replaced with (15), and its interpretation is that the only network edges on which the functional flux may flow are the ones on which power is flowing (i.e., $a_{i j}(h)=1$ ). Constraint (17) defines the centrality when active, and its activation is guaranteed by the minimization of $\mathcal{F}_{3}$, which, in turn, steers the system to select configurations in which nodes with high risk levels have low centrality, as a measure to avoid cascading failures. The considered centrality index is, then, proportional to the square of the number of descendant nodes that depend on node $i$, as it is proportional to the square of the amount of auxiliary flux that goes through it. Hence, $\mathcal{F}_{3}$ penalizes configurations that associate nodes subject to high risk levels to central positions.

It is worth noting that we decided to capture the topological centrality, as by design, we considered the number of descendant nodes at risk to be more relevant than the amount of load. With trivial modifications, this functional flux can be adapted to use cases in which the priority is given to configurations that consider the quantity of supported load as a centrality index.

e) Voltage and current limits:

$$
\begin{gathered}
V_{i}^{\min } \leq V_{i}(h) \leq V_{i}^{\max } \quad \forall i \in \mathcal{V} \\
I_{i j}(h) \leq I_{i j}^{\max } \quad \forall(i, j), i \geq j
\end{gathered}
$$

where $I_{i j}(h)$ is the line current magnitude, which can be written in function of voltages as shown in [4] (see (5)-(9) therein).

f) Step-voltage regulation at primary substations: The voltage level at the substation buses can be controlled by acting on the transformers' OLTC (see, e.g., [29]), which can be used to select different voltage levels

$$
\begin{aligned}
V_{i}(h) & =\sum_{j=1}^{l_{i}} \delta_{i j}(h) V_{i j}^{\mathrm{OLTC}} \quad \forall i \in \mathcal{V}^{\mathrm{SB}} \\
\sum_{j=1}^{l_{i}} \delta_{i j}(h) & =1 \quad \forall i \in \mathcal{V}^{\mathrm{SB}}
\end{aligned}
$$


where $l_{i}$ is the number of the voltage levels $V_{i j}^{\text {OLTC }}$ that can be selected at substation bus $i . \delta_{i j}(h)$ is a Boolean control variable used to select the voltage level. The second constraint assures that only one voltage level is selected at time $h$.

g) ESS constraints and dynamics: ESS constraints are introduced in order to model the ESS power rating, battery capacity, SOC dynamics; also a terminal constraint is introduced to guarantee system stability

$$
\begin{aligned}
P_{i}^{\mathrm{ESS}, \mathrm{min}} & \leq P_{i}^{\mathrm{ESS}}(h) \leq P_{i}^{\mathrm{ESS}, \text { max }} \\
Q_{i}^{\mathrm{ESS}, \min } & \leq Q_{i}^{\mathrm{ESS}}(h) \leq Q_{i}^{\mathrm{ESS}, \text { max }} \\
\operatorname{SOC}_{i}^{\text {min }} & \leq \operatorname{SOC}_{i}(h) \leq \mathrm{SOC}_{i}^{\text {max }} \quad \forall i \in \mathcal{V}^{\mathrm{ESS}} \\
\operatorname{SOC}_{i}(h+1) & =\operatorname{SOC}_{i}(h)-\mathrm{TP}_{i}^{\mathrm{ESS}}(h) \frac{100}{C_{i}^{\mathrm{ESS}}} \\
\operatorname{SOC}_{i}(k+N) & \geq \operatorname{SOC}_{i}^{\mathrm{ref}} .
\end{aligned}
$$

$T \in \mathbb{R}$ is the discretization time step. In relation to (23), notice that it is possible to act on the ESS inverter to control the reactive power. The reader is referred to the literature (see, e.g., [30]) for details on ESS reactive power control.

h) Initial conditions: The EMPC problem is solved at each time $k$ based on the following initial conditions:

$$
\begin{gathered}
\operatorname{SOC}_{i}(k)=\operatorname{SOC}_{i}^{k} \quad \forall i \\
a_{i j}(k)=a_{i j}^{k} \quad \forall i
\end{gathered}
$$

where $\mathrm{SOC}_{i}^{k}$ and $a_{i j}^{k}$ are, respectively, the measured ESS SOC level and the known network configuration at $k$.

The above problem is a nonconvex, mixed-integer nonlinear programming problem.

\section{A. Conification of the Problem Constraints}

Based on the procedure in [6], the above problem can be transformed into an equivalent, conified, quadratic programming problem having the same optimal solution. The constraints involved in this transformation are the ones related to power flow, in particular (2) and (3), that due to their nonlinearity increase the complexity of the problem. The transformation is outlined in the following; full details are in [6].

The following substitutions are made through the inclusion of the new auxiliary variables $u_{i}(h)$ for $i \in \mathcal{V}$ and $R_{i j}(h), T_{i j}(h)$ for $(i, j): a_{i j}=1$

$$
\begin{aligned}
u_{i}(h) & \leftarrow V_{i}(h)^{2} / \sqrt{2} \\
R_{i j}(h) & \leftarrow V_{i}(h) V_{j}(h) \cos \theta_{i j} \\
T_{i j}(h) & \leftarrow V_{i}(h) V_{j}(h) \sin \theta_{i j} .
\end{aligned}
$$

With these positions, the power flow equations (2) and (3) become

$$
\begin{aligned}
& P_{i j}(h)=a_{i j}(h)\left[-G_{i j} R_{i j}(h)+B_{i j} T_{i j}(h)+G_{i j} \sqrt{2} u_{i}(h)\right] \\
& Q_{i j}(h)=a_{i j}(h)\left[B_{i j} R_{i j}(h)+G_{i j} T_{i j}(h)-B_{i j} \sqrt{2} u_{i}(h)\right] .
\end{aligned}
$$

As explained in [4], (32) and (33) can be exactly linearized by introducing new Boolean variables $u_{i}^{i j}(h)$ for each line $(i, j)$ connected to the generic bus $i$ (in particular, variables $u_{i}^{i j}(h)$ are defined for $i \in \mathcal{V}, j \in \mathcal{V}$ and $\left.(i, j): a_{i j}=1\right)$. The following additional auxiliary constraints are introduced to force variables $u_{i}^{i j}(h)$ to be equal to zero when the line is disconnected and equal to $u_{i}(h)$ otherwise

$$
\begin{gathered}
0 \leq u_{i}^{i j}(h) \leq \frac{\left(V_{i}^{\max }\right)^{2}}{\sqrt{2}} a_{i j}(h) \\
0 \leq u_{i}(h)-u_{i}^{i j}(h) \leq \frac{\left(V_{i}^{\max }\right)^{2}}{\sqrt{2}}\left(1-a_{i j}(h)\right) \\
2 u_{i}^{i j}(h) u_{j}^{j i}(h) \geq R_{i j}(h)^{2}+T_{i j}(h)^{2}, R_{i j}(h) \geq 0
\end{gathered}
$$

Then, with the above positions, it can be verified that (32) and (33) can be written in a linear form as

$$
\begin{aligned}
& P_{i j}(h)=-G_{i j} R_{i j}(h)+B_{i j} T_{i j}(h)+G_{i j} \sqrt{(2)} u_{i}(h) \\
& Q_{i j}(h)=B_{i j} R_{i j}(h)+G_{i j} T_{i j}(h)-B_{i j} \sqrt{(}(2) u_{i}(h) .
\end{aligned}
$$

In fact, the reader can check that, when $a_{i j}(h)=1,(37)$ and (38) coincide with (32) and (33), because $u_{i}^{i j}(h)=u_{i}(h)$ due to (35). When $a_{i j}(h)=0$, then $u_{i}^{i j}(h)=0$ due to (34), and also $R_{i j}(h)$ and $T_{i j}(h)$ are equal to zero, because of (36); hence, it is recovered that $P_{i j}(h)=0$ and $Q_{i j}(h)=0$, as expected.

Departing from [4] and [6], further attention is needed to manage variables $u_{i}$ and $V_{i}$ for $i \in \mathcal{V}^{\mathrm{SB}}$, since voltages at substation buses are control variables that can assume only discrete values, as modeled in (20). Considering (29) and (20)

$$
u_{i}(h)=\frac{\left(\sum_{j=1}^{l_{i}} \delta_{i j}(h) V_{i j}^{\text {OLTC }}\right)^{2}}{\sqrt{2}} .
$$

Considering that $\delta_{i j}(h)^{2}=\delta_{i j}(h)$, and that $\delta_{i j}(h) \delta_{i k}(h)=0$ for $j \neq k$, because of (21), then (39) finally becomes

$$
u_{i}(h)=\frac{\sum_{j=1}^{l_{i}} \delta_{i j}(h)\left(V_{i j}^{\mathrm{OLTC}}\right)^{2}}{\sqrt{2}} .
$$

The next section summarizes the proposed control algorithm.

\section{B. Overall Formulation}

Algorithm 1 (EMPC risk-aware optimization of distribution grid). At each time $k=1,2, \ldots$ do (see Fig. 1).

1) Measure $x_{k}$, the current state of the controlled elements in the grid (open/close state of each switch and SOC of each ESS). Acquire external input values (load and generation forecasts, predicted risk values).

2) Solve $\mathbf{u}_{k}^{*}=\operatorname{argmin}\left\{\mathcal{F}_{N}\left(k, \mathbf{x}_{k}, \mathbf{u}_{k}\right)\right\}$ in (1) subject to: (2)(40). (Find the optimal topology configuration, the optimal position of the tap changers and the optimal ESS power injections over the time interval $[k, k+N-1]$.)

3) Apply to the system the first sample of the optimal control sequence $\mathbf{u}_{k}^{*}$.

4) Wait for the next sampling time and go to step 1). 


\section{Problem Complexity and Scalability of the Proposed Scheme}

The resulting problem is characterized by the presence of conic constraints and binary decision variables. Commercial solvers typically deal with such problems with variations of the branch and cut algorithm, meaning that the computational time required to find the optimal solution has an exponential dependence to the number of binary variables. Furthermore, it is shown in [6] that the solving time for the conified power flow problem on a fixed topology remains compatible with the application considered even in large networks (with a network of 88 nodes requiring less than twice the time of a 12 nodes one), supporting the fact that the main limiting factor of the proposed controller for scaling is related to the number of binary variables, and consequently to the number of available controllable switches.

In our formulation, each remote operable switch is associated with a decision variable for each time slot in the prediction, but in larger networks, one may assume to let the control variables $a_{i j}(h)$ change value only a given number of times in the prediction window, significantly reducing the number of binary variables of the problem (e.g., one reconfiguration allowed every hour). Furthermore, for testing purposes, we consider, in the following, a network whose links are all equipped with a controllable switch, while in real scenarios, the degree of freedom available to the controller is expected to be smaller, as only a portion of the network switches is remotely controllable. To control networks with a high number of controllable switches, a possible approach is that of computing offline the set of admissible configurations (which, for radiality, operative, and power-quality concerns, has a cardinality significantly lower than total number of possible configurations) and let the EMPC controller select a configuration only in this set. Doing so would significantly reduce complexity, since the number of binary variables would be significantly reduced and the constraints for radiality assurance could be removed. This option, combined with limiting the number of allowed switching in the prediction window, allows the solving time to be adequate to use cases of a larger scale than the ones considered in this article and in the H2020 Atena project.

\section{Simulation Results}

\section{A. Simulation Setup}

The proposed algorithm has been tested on a 16-bus threefeeder distribution network commonly employed in the literature (see Fig. 2) network data are taken from [32]. The network was chosen so that it presents a high number of potential configurations that differ significantly, allowing a clearer interpretation of the results.

In order to provide a proof of concept, common input data for all the use cases are provided, as follows. MV/LV substations placed along the feeders and serving end users are characterized by a typical summer load pattern [33], as reported in Fig. 3, while the MV busbars of the HV/MV substations are characterized by a small constant consumption of $10 \mathrm{~kW}$ related to the auxiliary devices; prediction and actual consumption patterns are assumed to be the same in the following. Three large-scale PV plants with $750 \mathrm{~kW}$ peak capacity are placed at nodes 4, 12, and 15;

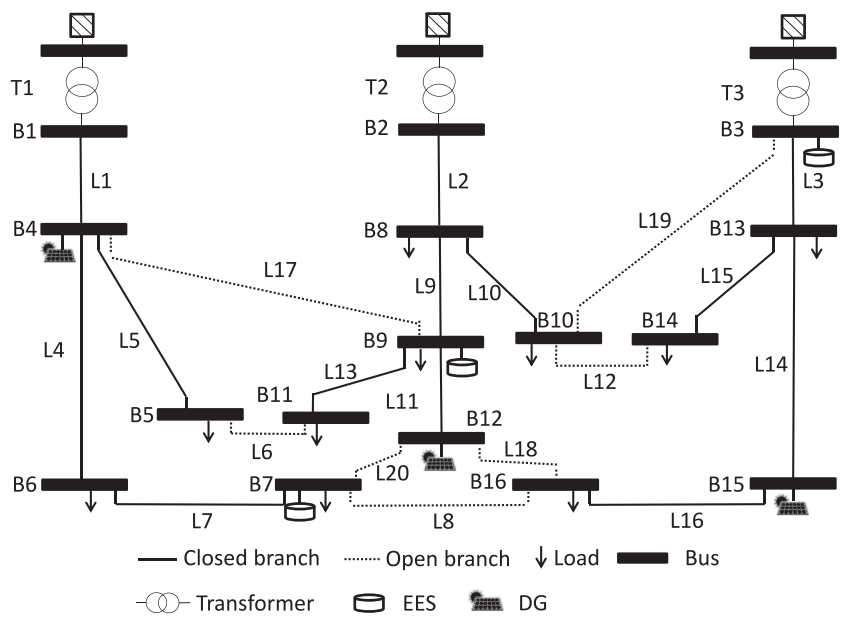

Fig. 2. Test distribution network (figure adapted from [31]). The benchmark configuration is the one with solid black lines.
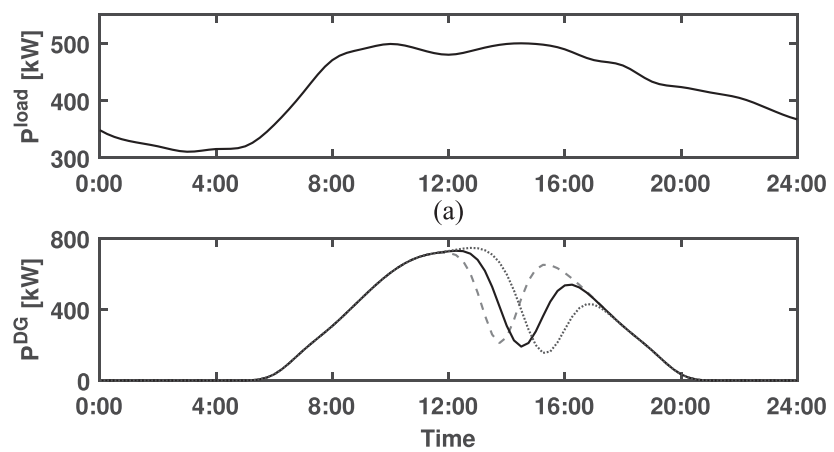

(b)

Fig. 3. (a) Power consumption at each of the MV/LV substations and (b) power generation at node 4 (dashed line), 12 (solid line), and 15 (dotted line). Notice the humps in the generation profiles, which are shifted in time in order to simulate a cloudy condition moving from the upper left portion of the network to the lower right one.

a typical clear sky pattern is initially considered as common day-ahead power prediction, and then perturbed in real time to build short-term power predictions that reconstruct the three actual generation patterns reported in Fig. 3(b). Three 2 MW/1 MVAr/2 MWh ESSs are placed at nodes 3, 7, and 9 to assess the effect of energy storing at different distances from the HV/MV substations; reference SOC levels have been set to $70 \%$ to give to the controller enough operational margin while storing enough energy to properly support the islanded operation of the system. The power factor is 0.98 for all the load and generation nodes, lagging or leading, respectively. The considered OLTCs possess 11 taps allowing $\pm 5 \%$ voltage variation with respect to the typical $20 \mathrm{kV}$ nominal level (the same range is imposed to all the nodes in the network). The problem was solved in per-unit to avoid numerical issues (solving optimization problems in which the variables can assume values over several different orders of magnitude may lead to numerical precision loss).

The sampling time $T$ and control horizon $N$ have been set to $15 \mathrm{~min}$ and $3 \mathrm{~h}$, respectively. The rationale behind the choice of $N$ is to take it large enough to provide flexibility to the system, while maintaining the number of variables and the complexity of the problem reasonable for a compatible solving 
time. Furthermore, a value of $N$ too large would introduce into the problem the high uncertainties associated with the predictions of the solar plant profiles and the risk levels far from the current time. Finally, the weights appearing in the target function have been empirically selected as $\alpha(h)=10, \gamma(h)=$ $1, \beta(h)=10^{-4}, \delta(h)=10^{-4}, \forall h$ in order to guarantee a good performance of the system in the various simulations. The parameter $\alpha$ was given the highest value in order to have the control focus more on power loss reduction, also taking care of the activation of constraint (35) and consequently assuring the correctness of the conification procedure. The weight $\gamma$ was given a secondary priority level. The weights relative to the control actions, namely, $\beta$ and $\delta$, were given a lower value in order to let the control system have a significant degree of freedom. In operative scenarios, the operator may desire to prevent a high number of reconfigurations, so a higher value could be given to $\beta$ or the variables $a_{i j}$ could be allowed to change values only at certain times (consequently lowering the problem complexity, as explained in Section III-C). More in general, the operator can carry out a detailed parameter selection study in order to best tune the system behavior according to its preferences, potentially by means of Monte Carlo simulations.

To establish a benchmark, Fig. 2 reports a configuration of the network with an approximately equal distribution of the load among the HV/MV substations, which reflects a typical practice adopted by distribution system operators for configuring the grid in absence of methods like the one here presented.

Simulations have been performed using the Julia v0.7 technical computing language, on an Intel I7, 8 GB RAM machine running Windows 10 . The optimization problem has been solved using Gurobi [34].

\section{B. Simulation 1: Minimization of Power Losses}

This simulation is aimed at assessing how the proposed control system manages the network losses, also taking into account RES fluctuations, assuming null risk level for all network components. Fig. 4(a) reports the comparison among the losses in the uncontrolled case (which considers the benchmark configuration) and according to the proposed EMPC. The losses in the controlled case always remain below the benchmark, with an energy saving at the end of the day of $23 \%$. Fig. 5 shows a significant subset of the 23 configurations taking place during the simulation. The configuration is the same during the hours when the generation is absent, while loads are connected as close as possible to the sources during the generation peak. At 14:30, the lowest point in bus 12 generation valley, the storage in bus 7 discharges feeding the largest tree, which is connected to the first HV/MV substation.

The ESSs at buses 7 and 9 basically behave as generators over the period in which the net load in the network has its peaks, and recharge in the valleys; the ESS at bus 3 is not activated, as expected, due to its placement in the HV/MV substation [see Fig. 4(b)]. The ESSs' SOC remains close to the reference, showing the feasibility of the ESSs contribution in the long term [see Fig. 4(c)]. Finally, Fig. 6 reports the voltages of the network buses over time. It can be seen that in the controlled scenario, the profiles remain similar to the uncontrolled one, with the voltages

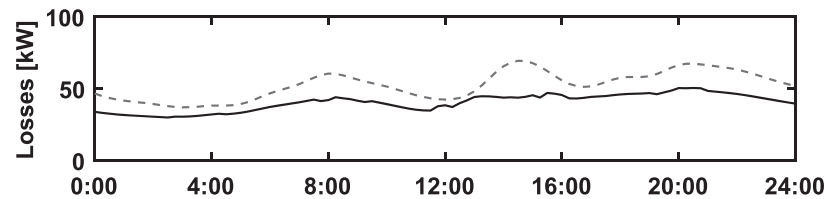

(a)

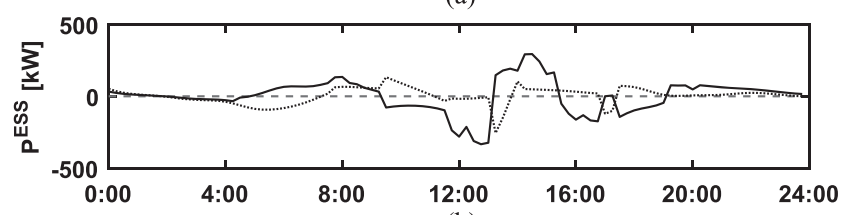

(b)

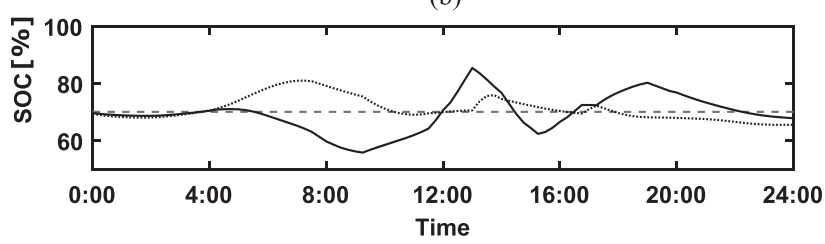

(c)

Fig. 4. Simulation 1. (a) Evolution of network power losses in the uncontrolled case (dashed line) and according to the proposed EMPC strategy (solid line), (b), (c) power and SOC of the ESSs installed at node 3 (dashed line), 7 (solid line), and 9 (dotted line).
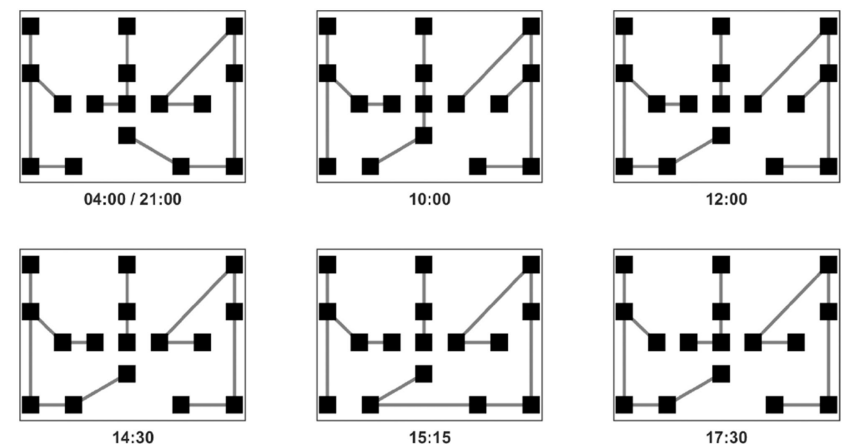

Fig. 5. Simulation 1: Network configurations at different times.

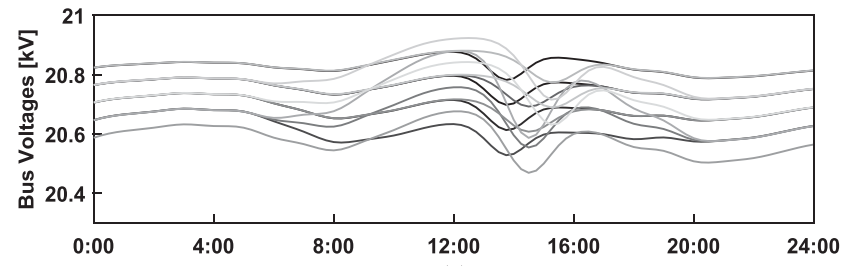

(a)

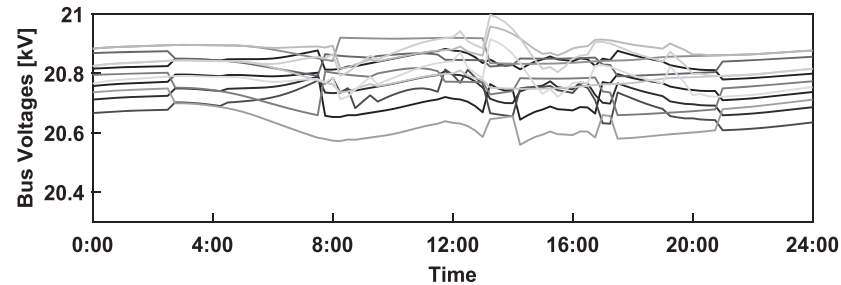

(b)

Fig. 6. Simulation 1. (a) Evolution of bus voltages in the uncontrolled case. (b) Evolution of bus voltages in the controlled case. 
TABLE I

Simulation 2: PREdicted COMPONENTS RISK LEVELS

\begin{tabular}{llll}
\hline Components & Risk Level & Start time & End Time \\
\hline Lines 10, 20 & $0.2 ; 0.2$ & $10: 45$ & $14: 45$ \\
Buses 9, 13 & $0.9 ; 0.8$ & $11: 45$ & $13: 45$ \\
\hline
\end{tabular}
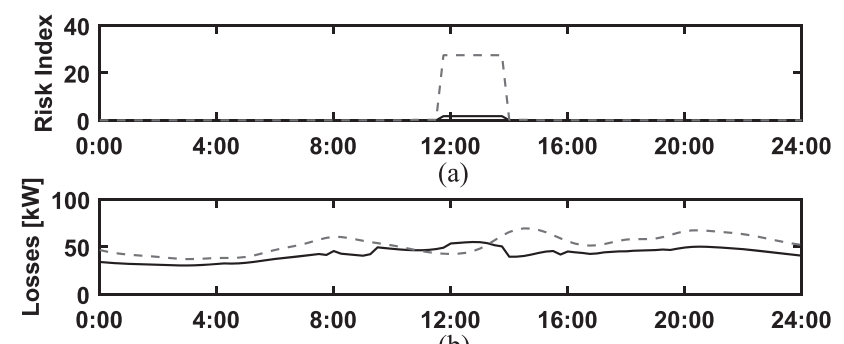

(b)

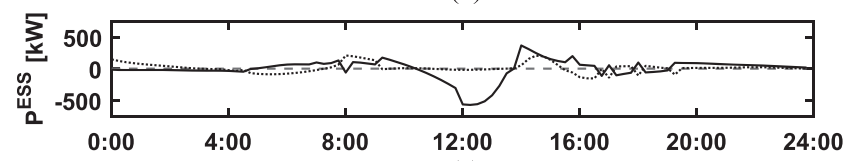

(c)

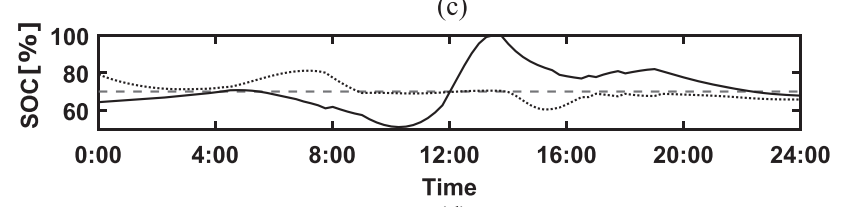

(d)

Fig. 7. Simulation 2. (a), (b) Evolution of network aggregated risk and power losses in the uncontrolled case (dashed line) and according to the proposed EMPC strategy (solid line). (c), (d) Power and SOC of the ESSs installed at node 3 (dashed line), 7 (solid line), and 9 (dotted line).

remaining in a slightly more contained region and the sudden changes in their profile caused by the reconfigurations. It can be noted that the OLTCs select the tap guaranteeing the maximum allowed voltage level $(21 \mathrm{kV})$ in all HV/MV substations, this allows us to minimize power losses and was possible for the absence of significant voltage increases over the other buses, as the power flow remained feasible for all times. Similar behavior is observed in the remaining simulations.

The average solving time of the EMPC iterations in this simulation was $79 \mathrm{~s}$, under the condition that the solution of the previous step was provided to the solver as an initial guess to speed up the convergence.

\section{Simulation 2: Preventive Mitigation of Adverse Events in the Distribution Grid}

In the following, it is shown how the proposed controller reacts to the notification of updated risk levels for network components, to simulate, e.g., attempts of attacks to switches or adverse natural events; the risk levels notified by the risk predictor are reported in Table I.

Fig. 7(a) and (b) reports the aggregated risk index, defined as $\mathcal{F}_{3}$ in Section III, and the power losses computed in the uncontrolled case and according to the proposed control. As expected, the overall risk level is mitigated, by $93 \%$, at the cost of reducing the performance in terms of energy saving (19\% reduction of losses, compared to previous 23\%). Note that, according to the definition given, the risk index is not normalized
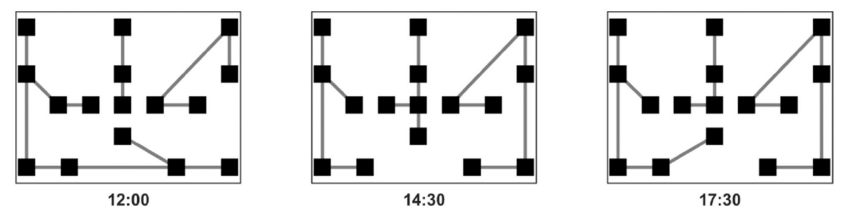

Fig. 8. Simulation 2: Network configurations at different times.

between 0 and 1 , and is instead a metric to differentiate two topologies based on the operative level, and positioning, of their nodes and links.

Fig. 8 presents configurations of the network computed respectively in instants in which the risk prediction is high on both nodes and links (12:00), only on links (14:30), and on neither (17:30). It can be seen that at 12:00, during the high risk time frame, the network is configured in such a way as to avoid the usage of both lines 10 and 20; also, the controller selects a configuration in which the buses 9 and 13 are at the end of the respective feeders, hence guaranteeing the power supply to consumers connected to those buses, while also avoiding cascading effects of a potential successful cyber-attack. Due to the asymmetric configuration characterized by a highly loaded tree departing from bus 1, all the generators are connected to that tree in order to mitigate the power losses; also the ESSs provide their additional contribution, see Fig. 7(c), basically amplifying the same behavior seen in Fig. 4(b). At 14:30, while still being provided with a high risk level for lines 10 and 20 , the controller selects a configuration more similar to the ones of the first simulation (see Section IV-B), since the risk prediction for the nodes is now set to zero. Finally, after the end of the alerting period, the configuration gradually returns the same one computed in absence of risk in Section IV-B (see the configuration at 17:30 in Fig. 8).

The average solving time of the EMPC iterations was $92 \mathrm{~s}$, higher compared to the previous simulation due to the presence of the constraints related to the centrality flux.

\section{Simulation 3: Preventive Mitigation of Adverse Events at Transmission/Distribution Interface}

The last simulation considers the opening of breakers in the HV/MV substations and the consequent disconnection of the distribution grid from the transmission network, which gives rise to controlled islanded operation starting from 11:30, a condition in which the balance between demand and supply is guaranteed by the ESSs. During islanding, the prediction window was shortened to $1 \mathrm{~h}$, to allow faster response of the system, and the network radiality was assured by constraint (6) and the power flow, as constraints (7)-(12) were no longer needed to guarantee the connection of the, now inactive, HV/MV substations to the storage devices. Also, the reference level for the SOC was lowered to $20 \%$ to encourage ESSs discharge at the beginning of the disconnection period to sustain the network, and consequently delay the shedding intervention. Fig. 9 shows how the proposed strategy keeps the most loads connected when the ESSs SOC is high, and then gradually increases the load shedding factor to prolong the survival time of the system. After 13:00, when the connection to the transmission is re-established, the ESSs recover their SOC at different speeds, according to their 


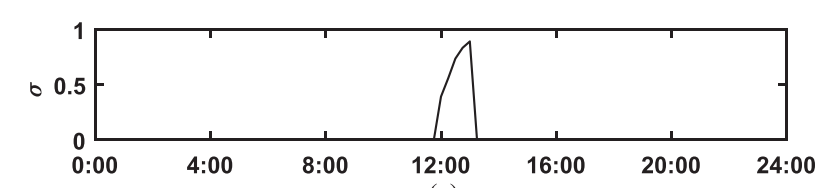

(a)

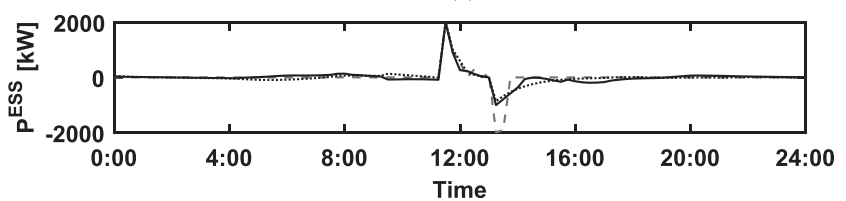

(b)

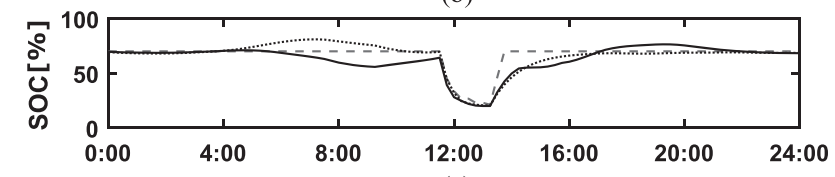

(c)

Fig. 9. Simulation 3. (a) Load shedding factor. (b), (c) Power and SOC of the ESSs installed at node 3 (dashed line), 7 (solid line), and 9 (dotted line).
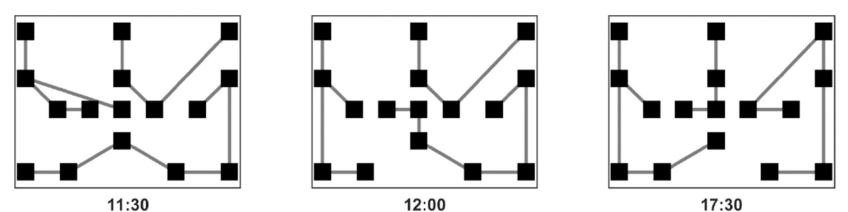

Fig. 10. Simulation 3. Network configurations at different sampling times during islanded operation.

topological position, in order to keep minimizing the network losses. Fig. 10 shows two configurations during the islanded operation, where the controller tries to balance the discharge of the ESSs by connecting most loads to the storage with the highest SOC, utilizing the generators to sustain the bigger trees. The third configuration reported shows that the same network configurations as the one in Section IV-B (normal operation) are recovered, after a transitory period during which the SOCs return close to their reference of $70 \%$. The average solving time was $64 \mathrm{~s}$.

\section{CONCLUSION}

This article presented a coordinated network reconfiguration and ESS MPC strategy for the efficient and secure operation of electricity distribution grids. The proposed approach enables the real-time reaction to the predictions of future fluctuations of power production from renewable and malicious/natural adverse events, with the aim of minimizing the network losses, the aggregated risk indicator, and allowing preventive or postattack grid operation.

The nonconvex optimization problem at the basis of the adopted MPC methodology incorporates the ESSs dynamics, the hybrid network power flow equations, and ad-hoc network topological constraints. The optimal solution of this problem is achieved at each sampling time by solving a conified proxy of the original problem, which has the same optimal solution. The proposed method is shown to be effective when applied to a 16-bus test distribution grid.

Future works will regard the investigation of the theoretical properties of the MPC algorithm, and a heuristic procedure for the identification of optimal values for the weighting terms in the objective function, starting from a high-level description of the operator preferences.

\section{ACKNOWLEDGMENT}

The authors gratefully acknowledge the members of the ATENA project and the CRAT team. The content of this article reflects only the author's view; the EU Commission is not responsible for any use that may be made of the information it contains.

\section{REFERENCES}

[1] A. K. Srivastava, A. A. Kumar, and N. N. Schulz, "Impact of distributed generations with energy storage devices on the electric grid," IEEE Syst. J., vol. 6, no. 1, pp. 110-117, Mar. 2012.

[2] W. Nwesaty, A. I. Bratcu, and O. Sename, "Power sources coordination through multivariable linear parameter-varying/ $h_{\infty}$ control with application to multi-source electric vehicles," IET Control Theory Appl., vol. 10, no. 16, pp. 2049-2059, 2016.

[3] C. Kwon and I. Hwang, "Cyber attack mitigation for cyber-physical systems: hybrid system approach to controller design," IET Control Theory Appl., vol. 10, no. 7, pp. 731-741, 2016.

[4] R. A. Jabr, R. Singh, and B.C. Pal, "Minimum loss network reconfiguration using mixed-integer convex programming," IEEE Trans. Power Syst., vol. 27, no. 2, pp. 1106-1115, May 2012.

[5] N. C. Koutsoukis, D. O. Siagkas, P. S. Georgilakis, and N. D. Hatziargyriou, "Online reconfiguration of active distribution networks for maximum integration of distributed generation," IEEE Trans. Autom. Sci. Eng., vol. 14, no. 2, pp. 437-448, Apr. 2017.

[6] R. A. Jabr, "Radial distribution load flow using conic programming," IEEE Trans. Power Syst., vol. 21, no. 3, pp. 1458-1459, Aug. 2006.

[7] Y. Liu, J. Li, and L. Wu, "Coordinated optimal network reconfiguration and voltage regulator/DER control for unbalanced distribution systems," IEEE Trans. Smart Grid, vol. 10, no. 3, pp. 2912-2922, May 2019.

[8] A. Di Giorgio, A. Giuseppi, F. Liberati, and A. Pietrabissa, "Controlled electricity distribution network black start with energy storage system support," in Proc. IEEE 25th Mediterranean Conf. Control Autom., 2017, pp. 781-786.

[9] M. N. B. Muhtazaruddin et al., "Distribution power loss minimization via distributed generation, capacitor and network reconfiguration," Indonesian J. Elect. Eng. Comput. Sci., vol. 5, no. 3, pp. 488-495, 2017.

[10] L. Bai, T. Jiang, F. Li, H. Chen, and X. Li, "Distributed energy storage planning in soft open point based active distribution networks incorporating network reconfiguration and dg reactive power capability," Appl. Energy, vol. 210, pp. 1082-1091, 2018.

[11] C. Lee, C. Liu, S. Mehrotra, and Z. Bie, "Robust distribution network reconfiguration," IEEE Trans. Smart Grid, vol. 6, no. 2, pp. 836-842, Mar. 2015.

[12] D.-L. Duan, X.-D. Ling, X.-Y. Wu, and B. Zhong, "Reconfiguration of distribution network for loss reduction and reliability improvement based on an enhanced genetic algorithm," Int. J. Electr. Power Energy Syst., vol. 64, pp. 88-95, 2015.

[13] L. Pfitscher, D. Bernardon, L. Canha, V. Montagner, V. Garcia, and A. Abaide, "Intelligent system for automatic reconfiguration of distribution network in real time," Electr. Power Syst. Res., vol. 97, pp. 84-92, 2013.

[14] H. Hijazi, C. Coffrin, and P. V. Hentenryck, "Convex quadratic relaxations for mixed-integer nonlinear programs in power systems," Math. Program. Comput., vol. 9, no. 3, pp. 321-367, Sep. 2017.

[15] S. F. Santos, D. Z. Fitiwi, M. R. Cruz, C. M. Cabrita, and J. P. Catalão, "Impacts of optimal energy storage deployment and network reconfiguration on renewable integration level in distribution systems," Appl. Energy, vol. 185 , pp. 44-55, 2017

[16] M. Lavorato, J. F. Franco, M. J. Rider, and R. Romero, "Imposing radiality constraints in distribution system optimization problems," IEEE Trans. Power Syst., vol. 27, no. 1, pp. 172-180, Feb. 2012.

[17] H. Ahmadi and J. R. Martí, "Mathematical representation of radiality constraint in distribution system reconfiguration problem," Int. J. Electr. Power Energy Syst., vol. 64, pp. 293-299, 2015.

[18] T. H. Chang, T. E. Lee, and C. H. Lin, "Distribution network reconfiguration for load balancing with a colored Petri net algorithm," in Proc. Int. Conf. Appl. Syst. Innov., May 2017, pp. 1040-1043. 
[19] T. T. Nguyen, T. T. Nguyen, A. V. Truong, Q. T. Nguyen, and T. A. Phung, "Multi-objective electric distribution network reconfiguration solution using runner-root algorithm," Appl. Soft Comput., vol. 52, pp. 93-108, 2017.

[20] M. R. M. Cruz, S. F. Santos, D. Z. Fitiwi, and J. P. S. Catalão, "Coordinated distribution network reconfiguration and distributed generation allocation via genetic algorithm," in Proc. IEEE Int. Conf. Environ. Electr. Eng. Ind. Commercial Power Syst. Eur., Jun. 2017, pp. 1-6.

[21] IEE, IEEE Guide for Electric Power Distribution Reliability Indices Redline, IEEE Std 1366-2012 (Revision of IEEE Std 1366-2003) - Redline, May 2012, pp. 1-92.

[22] D. Masucci, C. Foglietta, C. Palazzo, and S. Panzieri, "Improved multicriteria distribution network reconfiguration with information fusion," in Proc. 19th Int. Conf. Inf. Fusion, Jul. 2016, pp. 256-263.

[23] F. Adamsky et al., "Integrated protection of industrial control systems from cyber-attacks: The atena approach," Int. J. Critical Infrastructure Protection, vol. 21, pp. 72-82, 2018.

[24] X. Lu, N. Chen, Y. Wang, L. Qu, and J. Lai, "Distributed impulsive control for islanded microgrids with variable communication delays," IET Control Theory Appl., vol. 10, no. 14, pp. 1732-1739, 2016.

[25] M. Ellis, H. Durand, and P. D. Christofides, "A tutorial review of economic model predictive control methods," J. Process Control, vol. 24, no. 8, pp. 1156-1178, 2014.

[26] J. B. Rawlings, D. Angeli, and C. N. Bates, "Fundamentals of economic model predictive control," in Proc. IEEE 51st Conf. Decision Control, Dec. 2012, pp. 3851-3861.

[27] D. Mayne, J. Rawlings, C. Rao, and P. Scokaert, "Constrained model predictive control: Stability and optimality," Automatica, vol. 36, no. 6, pp. 789-814, 2000.

[28] P. Kundur, N. J. Balu, and M. G. Lauby, Power System Stability and Control. New York, NY, USA: McGraw-Hill, 1994, vol. 7.

[29] X. Liu, A. Aichhorn, L. Liu, and H. Li, "Coordinated control of distributed energy storage system with tap changer transformers for voltage rise mitigation under high photovoltaic penetration," IEEE Trans. Smart Grid, vol. 3, no. 2, pp. 897-906, Jun. 2012.

[30] S.-S. Shin, J.-S. Oh, S.-H. Jang, J.-H. Cha, and J.-E. Kim, "Active and reactive power control of ESS in distribution system for improvement of power smoothing control," J. Electr. Eng. Technol., vol. 12, no. 3, pp. 10071015,2017

[31] J. Pereira, J. Alves, and M. Matos, "Optimization of electrical distribution network operation based on EPSO," in Proc. 18th Int. Conf. Intell. Syst. Appl. Power Syst., Sep. 2015, pp. 1-6.

[32] S. Civanlar, J. J. Grainger, H. Yin, and S. S. H. Lee, "Distribution feeder reconfiguration for loss reduction," IEEE Trans. Power Del., vol. 3, no. 3, pp. 1217-1223, Jul. 1988.

[33] Terna s.p.a., "Terna s.p.a. web site," 2017. [Online]. Available: www.terna. it

[34] Gurobi Optimization, Inc., "Gurobi optimizer reference manual," 2016. [Online]. Available: http://www.gurobi.com

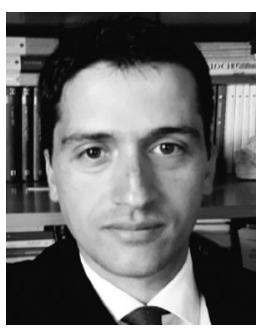

Francesco Liberati (Member, IEEE) was born in Rome, Italy, in 1987. He received the master's degree (summa cum laude) and the Ph.D. degree in systems engineering from the University of Rome, La Sapienza, Rome, Italy, in 2011 and 2015, respectively. From 2012 to 2017, he was a Researcher and Project Manager in automatic control and smart grids with CRAT, Rome, Italy. From 2015 to 2017, he was a Fixed-Term Researcher in automatic control with the eCampus University, Novedrate, Italy. In 2017 and 2018, he was a Project Manager with INEA, European Commission, Brussels, Belgium, managing smart grid and smart city H2020 research projects. He is currently a Researcher in automatic control with Sapienza University of Rome, Rome, Italy. His current research interests include model predictive control, learning in control, smart grids, and critical infrastructure protection.

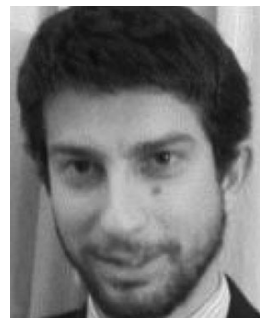

Alessandro Di Giorgio (Member, IEEE) was born on May 15, 1980, in Rome, Italy. He received the Laurea degree (cum laude) in physics in 2005, and the Ph.D. degree in systems engineering from the University of Rome, La Sapienza, Rome, Italy, in 2010.

$\mathrm{He}$ is currently an Associate Professor of automatic control, working on original applications of control systems theory to smart grids. He is author of about 60 papers and book chapters on these topics, mainly produced in the context of national and European research projects.

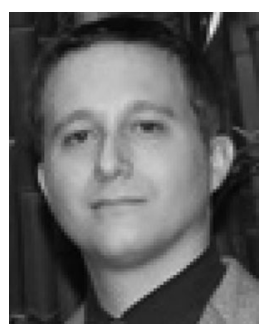

Alessandro Giuseppi (Member, IEEE) received the master's degree (summa cum laude) in control engineering from the University of Rome, La Sapienza, Rome, Italy, in 2016.

$\mathrm{He}$ is a Research Fellow with the Department of Computer, Control, and Management Engineering Antonio Ruberti, University of Rome, La Sapienza. Since 2016, he has participated in four EU projects, including H2020 ATENA, as a Researcher with CRAT, Rome, Italy. His main research interests include the application of control theory to smart networks and networked systems. He authored about 15 journal and conference papers on these topics.

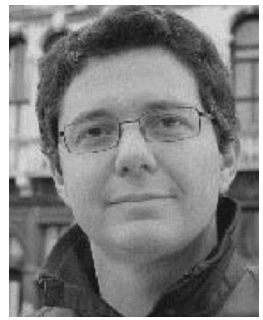

Antonio Pietrabissa (Member, IEEE) received the graduate degree in electronics engineering and the $\mathrm{Ph} . \mathrm{D}$. degree in systems engineering from the University of Rome, La Sapienza, Rome, Italy, in 2000 and 2004, respectively.

$\mathrm{He}$ is an Associate Professor with the Department of Computer, Control, and Management Engineering Antonio Ruberti, University of Rome, La Sapienza, where, since 2010 , he has been teaching automatic control and process automation. Since 2000, he has participated (either as Scientific Responsible or as Technical Coordinator) in about $20 \mathrm{EU}$ and National research projects. His main research interests include the application of systems and control theory methodologies to the analysis and control of networks, with specific interest to the design of resource management protocols. He is author of about 40 journal papers and 80 conference papers and book chapters on these topics.

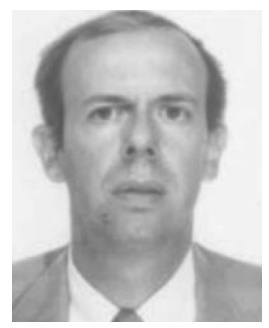

Francesco Delli Priscoli (Member, IEEE) was born in Rome, Italy, in 1962. He received the Graduate degree in electronics engineering (summa cum laude) and the Ph.D. degree in systems engineering from the University of Rome, La Sapienza, Rome, Italy, in 1986 and 1991, respectively.

From 1986 to 1991, he was with Telespazio, Rome, Italy. Since 1991, he has been with the University of Rome, La Sapienza, where, at present, he is a Full Professor of automatic control, control of autonomous multiagent systems, and control of communication and energy networks. His research interests include closed-loop multiagent learning techniques in advanced communication and energy networks.

Dr. Priscoli is an Associate Editor of Control Engineering Practice and a Member of the IFAC Technical Committee on Networked Systems. He was/is the Scientific Responsible with the University of Rome, La Sapienza, for 40 projects funded by the European Union and by the European Space Agency. 